
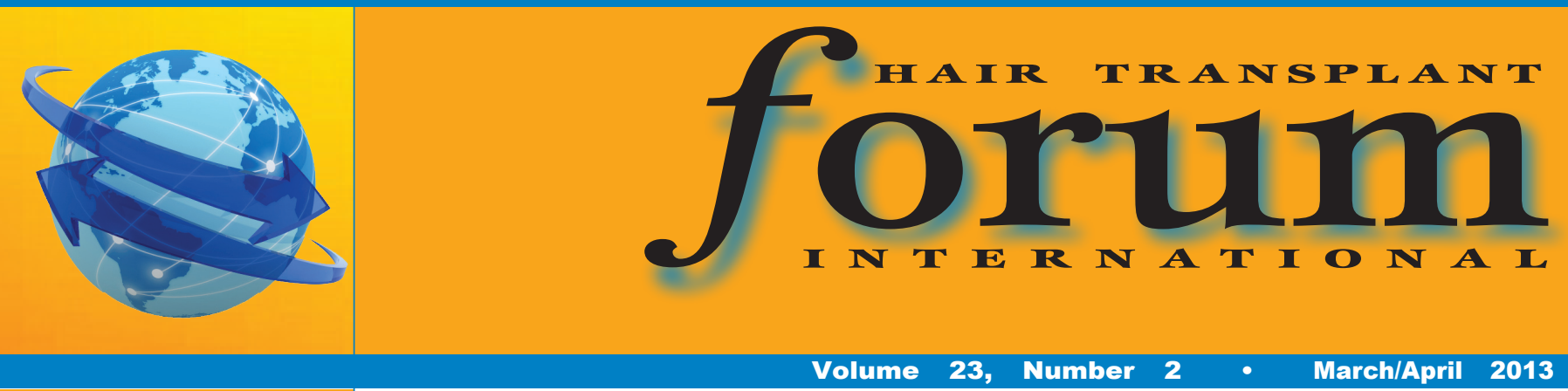

Inside this issue

President's Message

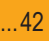

. .43

Co-editors' Messages

Notes from the Editor Emeritus:

Michael Beehner, MD.

Cell-based treatments for hair loss: research update on "hair cloning"..

Tissue expansion and hair restoration..

An overview of Biotin as it relates to hair loss...

Folliculitis and scarring after eyebrow transplantation: an uncommon complication.

Hair transplantation into scars.

Hair's the Question

How I Do It: Handy tips for slit depth control.

Cyberspace Chat: Topical minoxidil and association with cardiac arrhythmia...

Controversies: Does FUE change donor planning?

Review of the 4th Annual St. Louis University Workshop

Letters to the Editors

Message from the 2013 ASM

Program Chair

In fond memory of

Dr. Arturo Sandoval ..

SAC: Hair transplant surgery and continuous improvement of processes using Lean Healthcare methodologies

Message from the 2013 ASM

Surgical Assistants

Program Chair

Classified Ads...

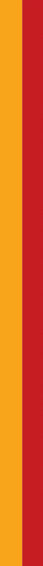

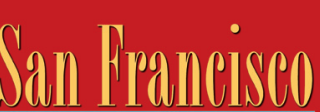

\section{Reflections on the role of hair grafting in wound healing}

\author{
Francisco Jimenez, MD Las Palmas, Spain fjimenez@clinicadelpelo.com
}

\section{Introduction}

Given the abundant body of evidence indicating that the main cellular machinery that fuels the wound healing process resides in hair follicles, why not use such machinery in clinical practice as a therapeutic weapon to stimulate the healing of chronic ulcers? This is the fundamental question that we tried to address in a paper recently published in the journal Wound Repair and Regeneration. ${ }^{1}$

\section{The Hair Follicle and the Healing Process}

The hair follicle appears to be the main anatomical structure that contributes to the healing process of cutaneous wounds. There is abundant clinical information to support the fact that wound healing starts around the hair follicles. Both the epithelial portion and the mesenchymal portion of the hair follicle contribute to the healing of skin wounds.

I would highly recommend a paper published in 1945 by G.H. Bishop, a neuroanatomist from Washington University in St. Louis. ${ }^{2}$ This paper, published in the American Journal of Anatomy, is the most relevant paper I have read about the wound healing process in humans. Bishop studied how the connective tissue and epithelium regenerate after removal of superficial layers of the skin. What is more, he did it in the hardest, but at the same time, the most objective way he could by self-inflicting cutaneous wounds on his forearm at different tissue depths and observing the healing process that took place clinically and histologically. He noted that not only did reepithelialization start around the remaining hair follicles, but also that when the skin was destroyed down to the deep dermis, the granulation tissue that regenerated came from the connective tissue surrounding the hair follicles.

Wounds in hairy areas heal faster than in non-hairy areas. One fact that supports the notion that hair follicles contribute to the healing process is the clinical evidence showing that wounds heal faster in hairy than in non-hairy zones. This property has been clinically demonstrated when scalp is the donor source for harvesting split-thickness grafts $(0.2-0.3 \mathrm{~mm}$ in depth): the healing time to complete reepithelialization of the donor wound averages 5 days in comparison with 10 days in areas such as the thighs, buttocks, or abdomen. ${ }^{3}$

The hair follicle is the main repository of cutaneous stem cells with multipotent capacity. At cellular level, the follicular stem cells are key players in the proliferation and differentiation of follicular cells in the wound bed. Keratinocytes that reepithelialize the wound are thought to derive from hair follicle epithelial stem cells that reside within the bulge. Hair follicle dermal papilla and dermal sheath harbor mesenchymal stem cells for which a role in wound healing has been proposed. Accordingly, transplantation of hair follicles with vital dye-labeled dermal sheath cells into a murine wound bed resulted in dermal sheath fibroblasts from the lower hair follicle being incorporated into the wounded dermis. ${ }^{4}$

\section{Why Transplant Hair Grafts in Chronic Leg Ulcers?}

Chronic leg ulcers are one of the most costly and prevalent diseases for health services worldwide. Have you ever considered how much money is spent on the treatment of a patient with a chronic leg ulcer? Accurate estimations recently published give a figure of 9,569 euros per ulcer per year. We are facing a major health care problem that consumes an enormous amount of highly costly resources. ${ }^{5}$ Most cutaneous chronic ulcers are located in the lower extremity and are caused by venous insufficiency, followed by arterial insufficiency and neuropathy (especially of diabetic etiology). It is estimated that 1 out of 100 adults will suffer from chronic leg venous ulcers at some point in their lifetime.

Chronic leg ulcers need better therapies. Compression therapy and wound dressings continue to be the mainstay treatment for venous ulceration in clinical practice. However, some $20 \%$ of ulcers remain unhealed after more than 50 weeks of appropriate compression therapy. Skin grafts have been used, but there is still insufficient evidence for the effectiveness of autologous skin grafting. ${ }^{6}$ Those grafts were taken from non-hair-bearing areas such as the buttocks that are preferentially composed of dermal tissue with no terminal hair follicles. By contrast, the original approach of our research work is that the punches are removed from the patient's scalp using a small punch, as is usually performed in hair transplantation (punch grafting technique). 
Hair grafting in wound healing

from page 41

\section{Pilot Study Results}

Ten patients with ulcers $(60 \%$ venous and $40 \%$ mixed) of an average $36.8 \mathrm{~cm}^{2}$ and a 10.5 -year duration were included in this pilot study. Within each ulcer we randomly assigned a $2 \times 2 \mathrm{~cm}$ "experimental square" to receive 20 hair grafts and a non-grafted "control" square of equal size. A transplant density of 5 follicular grafts $/ \mathrm{cm}^{2}$ was chosen as we considered this to be the minimum density required to guarantee tissue regeneration of the treated square of the ulcer.

Hair grafts were harvested from the occipital scalp using small-diameter (1-2mm) circular punches. After anesthetizing the "experimental square" of the wound bed, each punch hair graft was introduced one at a time using the "stick-and-place" method. The donor area was left to heal by secondary intention. Once the 20 hair grafts had been inserted in the experimental square, the whole ulcer was covered with Vaseline gauze and closed with an elastic bandage for 24 hours.

At the 18-week end point, we observed a $27 \%$ ulcer area reduction in the experimental square as compared with $6.5 \%$ in the control square $(p=0.048)$ with a maximum $33.5 \%$ vs. $9.7 \%$ reduction at week $4(p=0.007)$. Improvement of clinical symptoms (appearance of granulation tissue, wound border reactivation, and a lesser amount of exudation) was noted in 7 of the 10 patients.

\section{Conclusions and Research Implications}

This pilot study appears to indicate that hair grafting into chronic leg ulcers is not only practical but also successful in improving clinical evolution of ulcers of long duration.

This study was originally conceived as a feasibility trial. We did not address the various questions related to the hair grafting technique that are listed below:

- What is the ideal thickness of the punch ( $1 \mathrm{~mm}$ vs. $2 \mathrm{~mm}$, or even bigger)? We chose a size of punch that would not require suturing the donor wound but it didn't need to be a $1 \mathrm{~mm}$ punch since we were not concerned with the naturalness of the transplant. Our main aim was to develop a method without excessive technical difficulties regarding the extraction of entire hair follicles as would occur with a $2 \mathrm{~mm}$ punch.

- What would be the ideal recipient density in order to ensure intermesenchymal connections and to promote granulation tissue?

- Would it be better to insert the hair grafts closest to the border of the ulcer to take advantage of the "edge effect" from the reepithelialization border, or should we transplant the hair grafts randomly?

We do not know why 3 out of the 10 patients did not improve. We wondered if there are other factors that play a role in the success or failure of this technique such as infection of the wound bed, post-op care, etc.

We need to investigate the application of this technique in other non-venous leg ulcers such as diabetic, ischemic, etc. Would there be any influence of the etiology of the ulcers on the outcome of this therapy?
More studies are needed to compare hair grafting with simple dressings or to compare hair grafting obtained from the scalp versus skin grafts obtained from non-hairy areas. As hair transplant surgeons, we are in the perfect position to undertake this kind of investigation. Our field can expand enormously if we use our skills in other pathologies besides baldness.

\section{References}

1. Jimenez, F., et al. A pilot clinical study of hair grafting in chronic leg ulcers. Wound Rep Reg. 2012; 20:806-814.

2. Bishop, G.H. Regeneration after experimental removal of skin in man. Am J Anat. 1945; 76:153-181.

3. Weyandt, G.H., et al. Split-skin grafting from the scalp: the hidden advantage. Dermatol Surg. 2009; 35:1873-1879.

4. Gharzi, A., A.J. Reynolds, and C.A. Jahoda. Plasticity of hair follicle dermal cells in wound healing and induction. Exp Dermatol. 2003; 12:126-136.

5. Purwins, S., et al. Cost-of-illness of chronic leg ulcers in Germany. Int Wound J. 2010; 7:97-102.

6. Jones, J.E., and E.A. Nelson. Skin grafting for venous leg ulcers. Cochrane Database Syst Rev. 2007; (2):CD001737.
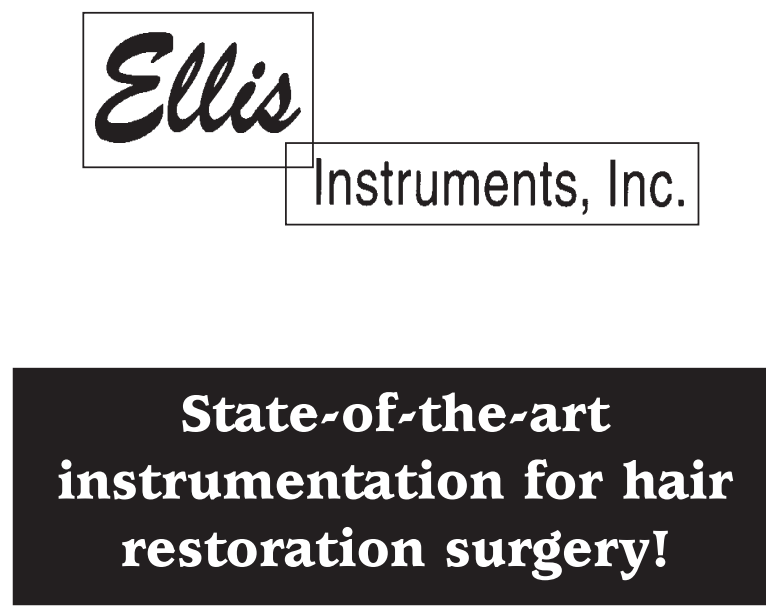

For more information, contact:

Phone: 800-218-9082 • 973-593-9222

Fax: 973-593-9277

E-mail: cellis@nac.net www.ellisinstruments.com 